\title{
Initiation of glucose-lowering therapy in Type 2 diabetes mellitus patients in general practice
}

\author{
J. A. Spoelstra*t, R. P. Stolk*, O. H. Klungelt, J. A. Erkens*†, G. E. H. M. Rutten*, \\ H. G. M. Leufkenst and D. E. Grobbee*
}

*Julius Center for Health Sciences and Primary Care, University Medical Center Utrecht and tDepartment of Pharmacoepidemiology and Pharmacotherapy, Utrecht Institute for Pharmaceutical Sciences (UIPS), Utrecht University, the Netherlands

Accepted 3 November 2003

\begin{abstract}
Aim The purpose of this study was to investigate which factors determine the initiation of glucose-lowering therapy in patients with Type 2 diabetes mellitus in general practice and their future glycaemic control.

Methods All incident Type 2 diabetic patients in the general practices in a Dutch middle-sized town from 1994 to 2000 were identified. Factors associated with initiation of glucose-lowering therapy were obtained from clinical files and examined by Cox's regression analyses. Using ANOVA, the associations between clinical characteristics at diagnosis and future glycaemic control were investigated.

Results In total, 603 newly diagnosed patients with Type 2 diabetes mellitus were included in the study. In the first month following diagnosis, $319(53 \%)$ started with oral therapy. One, two and three years after diagnosis of diabetes, the cumulative incidences were $71 \%$ (95\% CI 66-73\%), 75\% (71-79\%) and $81 \%$ (77-84\%), respectively. Age, gender, body weight, blood pressure, history of cardiovascular disease or total serum cholesterol values were not associated with time to start of drug therapy. An increased plasma glucose level at diagnosis was strongly related to faster initiation of drug therapy and worse future glycaemic control. Immediate initiation of glucose-lowering medication was not related to future glycaemic control.

Conclusion This study shows that the initial severity of diabetes, assessed by the degree of hyperglycaemia at time of diagnosis, is a major factor in determining the time to start of glucose-lowering drugs and the likelihood of achieving target levels of glycaemic control in the future, independent of glucose-lowering strategy. Therefore, patients with high glucose levels at diagnosis need close monitoring from the beginning of their disease.
\end{abstract}

Diabet. Med. 21, 896-900 (2004)

Keywords glucose-lowering treatment, newly diagnosed diabetes, glycaemic control

\section{Introduction}

Glycaemic control is the cornerstone of the management of Type 2 diabetes mellitus. Although difficult to achieve, modest weight loss and increased exercise have beneficial effects on

Correspondence to: Ronald P Stolk, Julius Center for Health Sciences and Primary Care, University Medical Center Utrecht, Hp D01.335, PO Box 85500, 3508 GA Utrecht, the Netherlands. E-mail: R.P.Stolk@jc.azu.nl glucose values, lipids and blood pressure [1-3]. When, despite implementation of these lifestyle changes, the fasting blood glucose target $(<8.0 \mathrm{mmol} / \mathrm{l})$ is not achieved within 3 months, drug treatment should be considered $[4,5]$.

Glycaemic control deteriorates gradually with time, even in intensively treated patients [6]. This worsening of glycaemic control has been attributed to the natural course of Type 2 diabetes and lack of efficacy of current hypoglycaemic therapy [6]. It has been shown that early addition of insulin or metformin 
can significantly improve glycaemic control without leading to increased hypoglycaemia or weight gain $[6,7]$.

However, little is known about the extent and effectiveness of lifestyle interventions (dietary treatment, increased exercise level) in primary care. For instance, in some cases doctors decide to start drug therapy immediately following diagnosis, but even in patients with high fasting glucose levels at diagnosis, a substantial proportion reach adequate metabolic control on lifestyle interventions or oral treatment without insulin [8]. The initiation of glucose-lowering medication is based on a combination of clinical characteristics $\left(\mathrm{HbA}_{1 \mathrm{c}}\right.$, age, etc.) and the interaction between the treating physician and the patient [4]. There are little data on the clinical grounds (patient characteristics) on which general practitioners (GPs) make this decision. Moreover it is not well known if this decision determines future glycaemic control.

The aim of this study was to investigate which patient-related factors determine the initiation of oral glucose-lowering therapy in newly diagnosed Type 2 diabetes mellitus patients in general practice and whether the timing of initiation is related to future glycaemic control.

\section{Patients and methods}

\section{Study setting}

This study was performed among patients who received comprehensive primary care from 17 GPs in a Dutch middle-sized town $(n=50574)$. Detailed clinical information was captured in a single electronic medical record system (Medicom $\AA$ ), whereas information on pharmacy-based drug dispensings was registered in a second database (Pharmacom®). Hospital admission and discharge data were available through the PHARMO Record Linkage System $[9,10]$. The following data were available for this study: demographic data, medical history, comorbidity [including International Classification of Primary Care (ICPC) codes], diabetic complications, laboratory results, prescriptions and drug dispensings, doctor in attendance (specialist, GP), referrals to specialists and a 'medical journal' (a database-file containing free text, as recorded by the GP in the computer).

To guarantee privacy, all analyses were performed using anonymous records. Regarding medication prescriptions and dispensings, all drugs were coded according to the Anatomical Therapeutic Chemical (ATC) Classification. Hospital diagnoses were coded according to the International Classification of Diseases, Ninth Revision, Clinical Modification (ICD-9-CM codes).

\section{Subjects}

In the Netherlands, most patients with diabetes mellitus Type 2 visit their GP for regular check-ups. They were identified from the registries by International Classification of Primary Care (ICPC-2) codes T90 or T90.2 and/or the description 'diabetes mellitus Type 2' in their medical records. Data from both primary care and the pharmacy-based dispensing records were complete for the period of February 1994 to August 2000 and were considered for the present study.

For the present study, we selected all newly diagnosed patients with Type 2 diabetes during this period $(n=603)$. Incident cases were defined as diabetes patients with no previous mention of diabetes in the GP file and no preceding glucoselowering medication use. Following the Dutch General Practitioners' Guidelines, treatment of newly diagnosed patients starts with dietary advice and encouragement of physical activity [5]. Oral glucose-lowering medication is indicated if target levels of blood glucose are not achieved within 3 months. Glycaemic control was defined in terms of poor $\left(\mathrm{HbA}_{1 \mathrm{c}}>8.5 \%\right)$, acceptable $\left(\mathrm{HbA}_{1 \mathrm{c}} 7.0-8.5 \%\right)$ and good control $\left(\mathrm{HbA}_{1 \mathrm{c}}<7.0 \%\right)$ according to the College of Dutch General Practitioners' Guidelines [5].

\section{Data analysis}

For categorical variables, numbers and percentages and for continuous data means and standard deviations (SD) or standard errors (SE) were calculated. For comparison of continuous variables and categorical variables, we used the Students' $t$-test and $\chi^{2}$-test, respectively. The Kaplan-Meier method was used to calculate the cumulative incidence of glucose-lowering drug use, under the assumption that treatment will be continuous after initiation of this medical therapy. Log rank tests were performed to assess differences between subgroups.

We compared time to start of oral hypoglycaemic drug treatment over four quartiles of fasting and non-fasting blood glucose levels at diagnosis (FBG and NFBG), using linear regression. Spearman's correlation coefficient was calculated to study the association between FBG and NFBG levels.

Furthermore, we used Cox's proportional hazards analyses to investigate the associations between initial glucose values and start of oral hypoglycaemic therapy. Date of censoring was the end of follow-up (death, migration, end of study in August 2000); co-variates included age and gender.

With respect to glycaemic control, mean differences between FBG and NFBG strata were analysed using analysis of variance (ANOVA), adjusted for potential confounders (age, gender and duration of diabetes). All analyses were carried out using the statistical package SPSS version 9.0 for Windows (SPSS Inc., Chicago, IL, USA).

\section{Results}

Table 1 shows the general characteristics of the 603 newly diagnosed patients included in this study. In total, 136 patients $(23 \%)$ remained on dietary treatment only (mean duration of diabetes 2.1 (sE 0.1 ) years and $66(11 \%)$ patients switched to insulin therapy after a mean duration of $1.6(0.2)$ years.

\section{Start of hypoglycaemic therapy}

Figure 1 represents the cumulative incidence (Kaplan-Meier curve estimate) of glucose-lowering drug use in newly diagnosed Type 2 diabetes mellitus patients. Of the incident Type 2 patients, $319(53 \%)$ started immediately (in the first month following 


\begin{tabular}{|c|c|c|}
\hline Characteristic & Mean (SD) or \% & Total number of patients \\
\hline Age at onset (years) & $62.0(13.5)$ & $603(100 \%)$ \\
\hline Male (\%) & 43.4 & 262 \\
\hline Diabetes duration* (years) & $3.0(1.9)$ & $603(100 \%)$ \\
\hline $\mathrm{HbA}_{1 \mathrm{c}}(\%)$ & $7.6(1.5)$ & $317(53 \%)$ \\
\hline Weight (kg) & $83.8(17.8)$ & $303(50 \%)$ \\
\hline Body mass index $\left(\mathrm{kg} / \mathrm{m}^{2}\right)$ & $28.9(5.2)$ & $147(24 \%)$ \\
\hline Fasting blood glucose (mmol/l) & $8.7(2.5)$ & $444(74 \%)$ \\
\hline Random blood glucose $(\mathrm{mmol} / \mathrm{l})$ & $10.7(3.8)$ & $445(74 \%)$ \\
\hline Systolic blood pressure (mmHg) & $150.7(20.6)$ & $407(67 \%)$ \\
\hline Diastolic blood pressure $(\mathrm{mmHg})$ & $86.4(10.5)$ & $405(67 \%)$ \\
\hline Total serum cholesterol (mmol/l) & $5.7(1.0)$ & $373(62 \%)$ \\
\hline HDL cholesterol $(\mathrm{mmol} / \mathrm{l})$ & $1.20(0.31)$ & $265(44 \%)$ \\
\hline Ratio HDL/total cholesterol & $5.2(1.5)$ & $249(41 \%)$ \\
\hline LDL cholesterol (mmol/l) & $3.60(0.95)$ & $178(30 \%)$ \\
\hline Triglycerides (mmol/l) & $2.54(2.34)$ & $265(44 \%)$ \\
\hline History of cardiovascular disease $(\%)$ & 21.2 & 127 \\
\hline Switchers to insulin therapy $(\%)$ & 10.9 & 66 \\
\hline
\end{tabular}

Values are proportions or means with standard deviation between parentheses. Metabolic variables represent the average during the follow-up period.

*At date of end of study (31 August 2000) or date of censoring (loss to follow up).
Table 1 General characteristics of newly diagnosed patients with Type $2 \mathrm{DM}(n=603)$

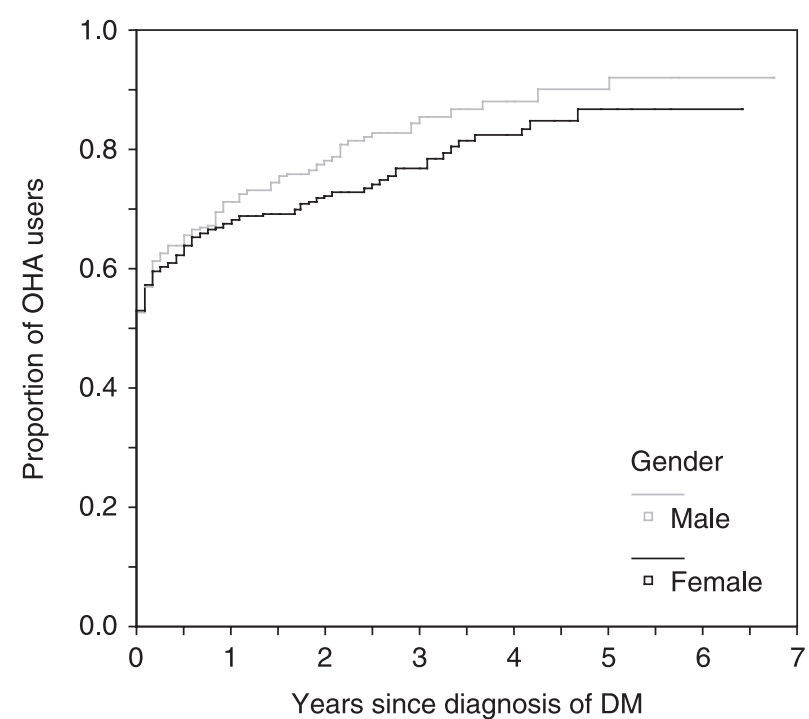

Figure 1 Start of oral hypoglycaemic agent use after diagnosis of diabetes.

diagnosis) with oral therapy. One, two and three years after diagnosis of diabetes, the cumulative incidences were $71 \%(95 \%$ CI $66-73 \%), 75 \%(71-79 \%)$ and $81 \%(77-84 \%)$, respectively. The curves show that men tended to start with drug treatment sooner after diagnosis, but this difference was not statistically significant ( $\log$ rank test statistic: $1.93, P=0.17$ ). Indeed, 3 years after diagnosis $21 \%$ of women and $15 \%$ of men were still on dietary treatment only. In more than half of the patients $(51.8 \%)$, tolbutamide was the drug of first choice, followed by a second-generation sulphonylurea (gliclazide, glibenclamide, glimepiride and glipizide; $30.4 \%$ ) and metformin $(18.2 \%)$. Acarbose was prescribed in only 10 patients $(2.1 \%)$ and 13 patients $(2.8 \%)$ started oral treatment with two drugs simultaneously, predominantly a combination of metformin and a sulphonylurea.

Age, body weight (sex-adjusted), systolic and diastolic blood pressure, history of cardiovascular disease or total serum cholesterol values were not associated with time to start of oral drug therapy. Mean fasting and/or non-fasting blood glucose (FBG and NFBG) at diagnosis were 10.8 (sD 4.0) and 14.6 (5.8), respectively. As shown in Fig. 2( $\mathrm{a}$ and b), initiation of hypoglycaemic drug therapy was strongly related to glucose level at diagnosis.

Initiation of insulin therapy was not related to glucose levels at diagnosis. In those patients who did switch to insulin, the time to treatment with insulin shortened with increasing blood glucose level, from 2.9 years (NFBG $<11.4 \mathrm{mmol} / \mathrm{l}$ ) to 1.3 years (NFBG > $16.2 \mathrm{mmol} / \mathrm{l}$ ); test for trend, $P=0.05$, adjusted for age and gender.

Time to first treatment with any oral hypoglycaemic drug increased from 0.2 years (SD 0.6) in those patients with a fasting plasma glucose $>13.1 \mathrm{mmol} / \mathrm{l}$ at the time of diagnosis to 1.4 years (SD 1.5 ) in patients with glucose $<7.9 \mathrm{mmol} / 1$ when their diabetes was diagnosed (test for trend $P<0.001$ ). In a Cox's regression analysis, adjusted for age at onset of diabetes and gender, this relationship remained statistically significant; Hazard Ratios were 1.7 (2nd quartile), 2.2 (3rd) and 2.9 (4th), respectively, compared with the group with lowest FBG values.

\section{Future glycaemic control}

Figure 3 shows the association between level of hyperglycaemia at diagnosis, according to quartiles of fasting blood glucose values and future glycaemic control (most recent $\mathrm{HbA}_{1 \mathrm{c}}$ 
(a)

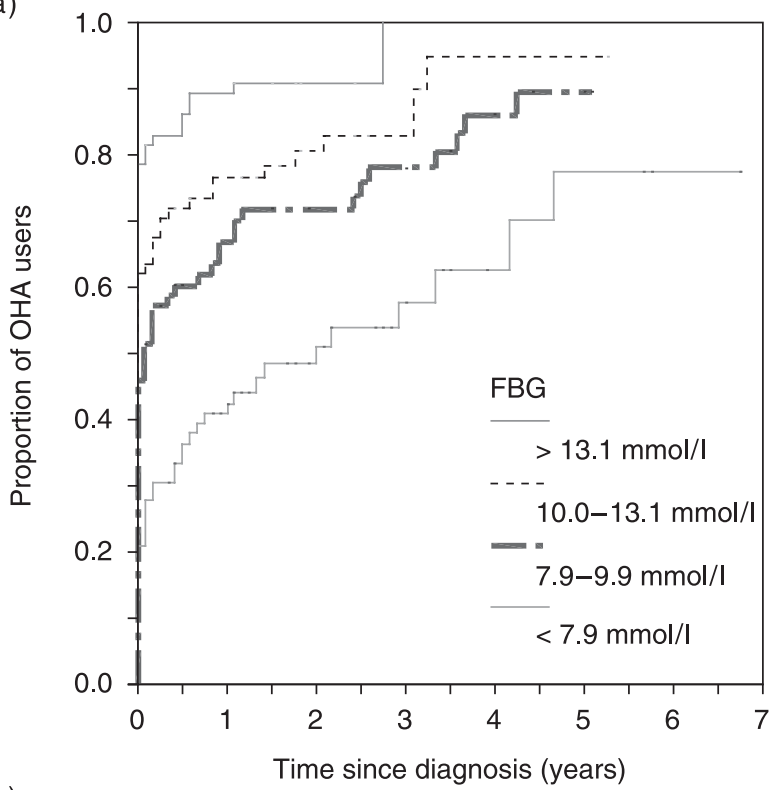

(b)

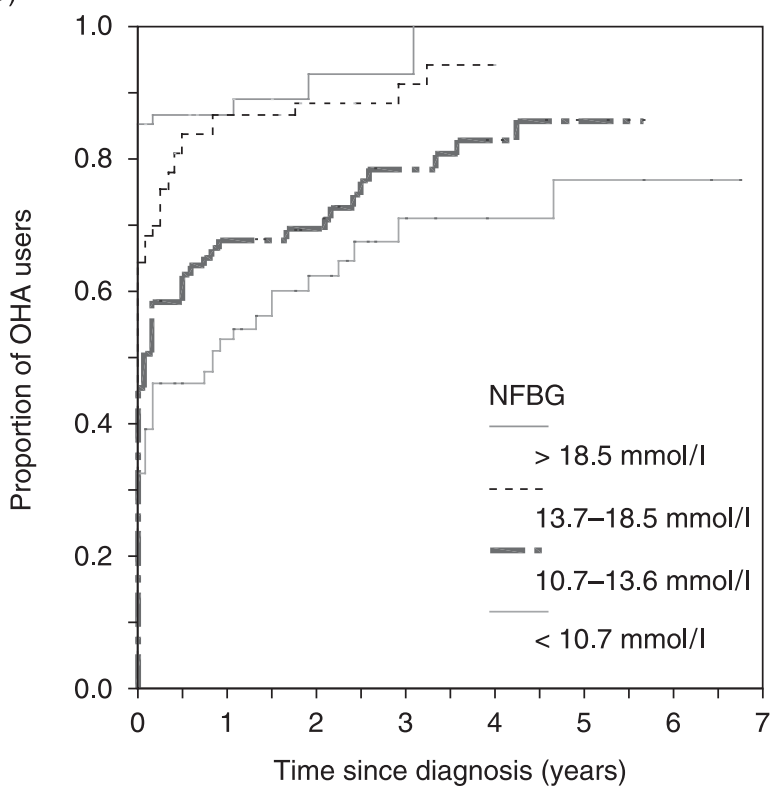

Figure 2 Start of oral hypoglycaemic therapy and glucose levels at diagnosis. (a) Fasting blood glucose and time-to-drug treatment. (b) Non-fasting blood glucose and time-to-drug treatment.

measurement available). The association between NFBG at diagnosis and actual glycaemic control followed a similar pattern (data not shown). Current glycaemic control differed significantly between quartiles of blood glucose values at diagnosis $(P=0.016$, adjusted for age, gender and duration of diabetes). The mean duration of diabetes at the time of this measurement was 2.4 years and did not differ between quartiles.

In patients with similar glucose levels at diagnosis (defined by tertiles of FBG and NFBG levels), immediate initiation of glucose-lowering medication was not significantly related to future glycaemic control.

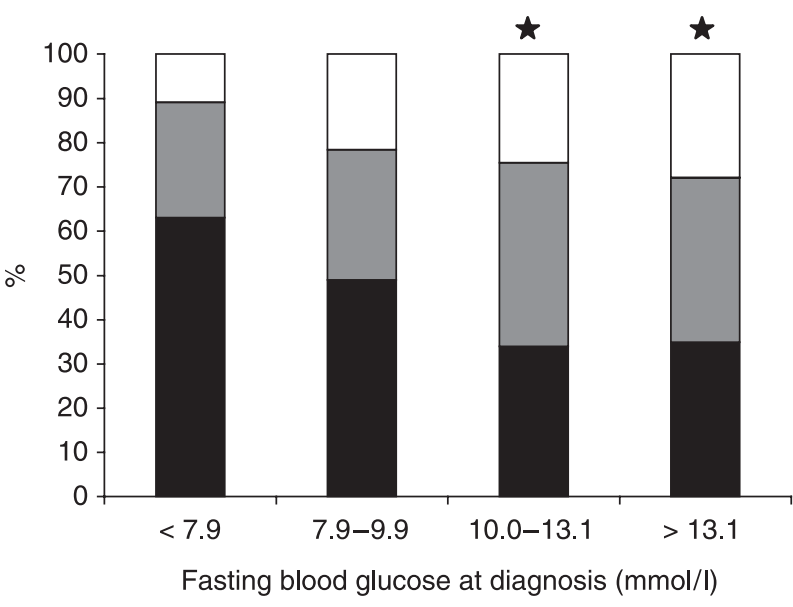

Figure 3 Glucose level at diagnosis and future glycaemic control. ${ }^{*} P<0.01$, metabolic control compared with the first quartile. Definition of glycaemic control: $(\square)$ poor, $\mathrm{HbA}_{1 \mathrm{c}}>8.5 \%$; $(\square)$ acceptable, $\mathrm{HbA}_{1 \mathrm{c}}$ between 7.0 and $8.5 \%$; ( $)$ good, $\mathrm{HbA}_{1 \mathrm{c}}<7.0 \%$.

\section{Discussion}

We assessed biomedical determinants of initiation of pharmacological glucose-lowering treatment in newly diagnosed Type 2 diabetes mellitus patients in general practice. Fiftythree per cent of the patients started immediately with blood glucose-lowering therapy. The strongest predictors of time-todrug therapy were fasting and non-fasting blood glucose levels at diagnosis. Also, a tendency to prescribe oral hypoglycaemic agents sooner in men than women was shown, although not statistically significant. Furthermore, blood glucose levels at diagnosis of diabetes predicted future glycaemic control; patients in the lowest quartiles had significantly lower future $\mathrm{HbA}_{1 \mathrm{c}}$ levels, independent of actual glucose-lowering therapy.

In the United Kingdom Prospective Diabetes Study, higher glucose level at diagnosis was also associated with poorer prognosis: patients with high glucose levels suffered from more subsequent cardiovascular disease, ischaemic heart disease in particular, retinopathy, erectile dysfunction, and showed progressive requirement for multiple therapies [11-13].

The strength of this study is the use of routinely collected primary care data, which reflect usual clinical care. General practice networks provide databases that provide useful research material. The use of computerized databases permits analyses of diagnostic, treatment and prescribing patterns in different patient groups within the general population. These databases enhance access to health-related information of large groups of patients over a long period of time $[14,15]$. From other studies, it is known that the sensitivity of general practice registries in identifying patients with diagnosed diabetes exceeds $90 \%$ [16].

Some limitations of this study need to be addressed. In only $66 \%(398 / 603)$ of the patients was a blood glucose value at diagnosis recorded in their medical notes. This can partly be explained by the fact that many patients are diagnosed by an 
accidental finding of increased glucose levels in routine laboratory examinations performed in hospitalized patients. In other patients, the GP might not have noted the result of a capillary glucose measurement performed in the practice. Furthermore, data on body weight and notably height were scarce.

According to the Dutch guidelines for Type 2 diabetes, pharmacological treatment has to be considered if target levels of glycaemic control are not achieved after dietary treatment period for at least 3 months [6]. However, the results study showed that in about half of the patients GPs started drug treatment immediately following diagnosis. Although patients who started with hypoglycaemic drugs immediately had higher glucose levels at diagnosis, GPs reasons for deviating from standard guidelines remain unclear. The presence (or absence) of severe symptoms may play a role in making this decision. However, we did not record patients' symptoms. Lack of financial support and insufficient dietitians may also be important issues.

Hyperglycaemia should be treated more aggressively in patients with a worse cardiovascular profile or established cardiovascular disease when diagnosing diabetes. However, we found time to start of oral glucose-lowering therapy was not associated with a history of cardiovascular disease and cardiovascular risk factors such as body weight, blood pressure and total serum cholesterol. This may reflect lack of awareness among GPs that diabetes is a multifactorial disease. Alternatively, current targets for glycaemia, lipids and blood pressure are attainable in only $50-70 \%$ of individuals with Type 2 diabetes [17].

We were intrigued by the observation that a small proportion (about $17 \%$ after 3 years of diabetes) of the patients remained on dietary treatment for long periods and achieved good glycaemic control $\left(\mathrm{HbA}_{1 \mathrm{c}}<7.0 \%\right)$. In the UKPDS, those patients were termed 'diet satisfactory' and excluded from the analysis after the 3-month run-in period. Of the initially 4075 included newly diagnosed Type 2 diabetes patients, after 3 years 357 $(11.5 \%)$ patients and even after 9 years $115(8.6 \%)$ patients maintained fasting plasma glucose levels $<6.0 \mathrm{mmol} / \mathrm{l}$ on diet only [11]. The most likely explanation for this observation is that Type 2 diabetes may be curable in moderately obese patients who achieve sufficient weight loss [18-20]. Alternatively, some patients may have been incorrectly diagnosed as having Type 2 diabetes mellitus.

In conclusion, our results show that initial severity of diabetes, assessed by the degree of hyperglycaemia at the time of diagnosis, is a major factor in determining the time to start of pharmacological treatment and the likelihood of achieving target levels of glycaemic control in the future, independent of immediate initiation of glucose-lowering medication. Patients with high glucose levels at diagnosis need close monitoring from diagnosis.

\section{Acknowledgements}

This study was supported by an unrestricted Novo Nordisk Fellowship, Alphen aan den Rijn, the Netherlands.

\section{References}

1 Markovic TP, Jenkins AB, Campbell LV, Furler SM, Kraegen EW, Chisholm DJ. The determinants of glycemic responses to diet restriction and weight loss in obesity and NIDDM. Diabetes Care 1998;21: 687-694.

2 Heilbronn LK, Noakes M, Clifton P. Effect of energy restriction, weight loss, and diet composition on plasma lipids and glucose in patients with type 2 diabetes. Diabetes Care 1999; 22: 889-895.

3 Williams KV, Mullen ML, Kelley DE, Wing RR. The effect of short periods of caloric restriction on weight loss and glycemic control in type 2 diabetes. Diabetes Care 1998; 21:2-8.

4 De Sonnaville JJJ, Heine RJ. Non-insulin dependent diabetes mellitus: presentation and treatment. Medicine 1997; 76: 23-26.

5 Rutten GEHM, Verhoeven S, Heine RJ, De Grauw WJC, Cromme PVM, Reenders K et al. Dutch College of General Practitioners' guidelines on type 2 diabetes mellitus (first revision). Huisarts Wet 1999; 42: 67-84.

6 UKPDS Group. Intensive blood-glucose control with sulphonylureas or insulin compared with conventional treatment and risk of complications in patients with type 2 diabetes (UKPDS 33). Lancet 1998; 352: 837-853.

7 Wright A, Burden ACF, Paisey RB, Cull CA, Holman RR for the UKPDS Group. Sulfonylurea inadequacy. Efficacy of addition of insulin over 6 years in patients with type 2 diabetes in the UK Prospective Diabetes Study (UKPDS 57). Diabetes Care 2002; 25: 330-336.

8 UKPDS Group. UK Prospective Diabetes Study 7: response of fasting plasma glucose to diet therapy in newly presenting type II diabetic patients. Metabolism 1990; 39: 905-912.

9 Herings RMC, Bakker A, Stricker BHC, Nap G. Pharmaco-morbidity linkage: a feasibility study comparing morbidity in two pharmacy based exposure cohorts. J Epidemiol Community Health 1992; 46: 136-140.

10 Herings RMC, De Boer A, Stricker BHCh, Leufkens HGM, Porsius A. Hypoglycaemia associated with use of inhibitors of angiotensin converting enzyme. Lancet 1995; 345: 1195-1198.

11 Turner RC, Cull CA, Frighi V, Holman RR. Glycemic control with diet, sulfonylurea, metformin, or insulin in patients with type 2 diabetes mellitus (UKPDS 49). JAMA 1999; 281: 2005-2012.

12 UKPDS Group. Hyperglycemia and hyperinsulinemia at diagnosis of diabetes and their association with subsequent cardiovascular disease in the United Kingdom Prospective Diabetes Study (UKPDS 47). Am Heart J 1999; 138: S353-359.

13 Colagiuri S, Cull CA, Holman RR for the UKPDS Group. Are lower fasting plasma glucose levels at diagnosis of type 2 diabetes associated with improved outcomes (UKPDS 61)? Diabetes Care 2002; 25: 1410-1417.

14 Glauber HS, Brown JB. Use of health maintenance organization databases to study pharmacy resource usage in diabetes mellitus. Diabetes Care 1992; 15: 870-876.

15 Metsemakers JFM, Höppener P, Knottnerus JA, Kocken RJJ, Limonard CBG. Computerized health information in the Netherlands: a registration network of family practices. $\mathrm{Br} \mathrm{J}$ General Pract 1992; 42: 102-106.

16 Morris AD, Boyle DIR, MacAlpine R, Emslie-Smith A, Jung RT, Newton RW et al. The diabetes audit and research in Tayside Scotland (DARTS) study: electronic database linkage to create a diabetes register. BMJ 1997; 315: 524-528.

17 Winocour PH. Effective diabetes care: a need for realistic targets. BMJ 2002; 324: 1577-1580.

18 Dixon JB, O’Brien PE. Health outcomes of severely obese type 2 diabetic subjects 1 year after laparoscopic adjustable gastric banding. Diabetes Care 2002; 25: 358-363.

19 Tayek JA. Is weight loss a cure for type 2 diabetes? Diabetes Care 2002; 25: 397-398.

20 Sjöström CD, Peltonen M, Wedel H, Sjöström L. Differentiated longterm effects of intentional weight loss on diabetes and hypertension. Hypertension 2000; 36: 20-25. 